\title{
Availability Analysis with Opportunistic Maintenance of a Two Component Deteriorating System
}

\author{
Joel P. Varghese and Girish Kumar
}

\begin{abstract}
This paper describes the opportunistic maintenance model for availability analysis of two component series system using the Markov and the Monte Carlo Simulation methods. The system model is developed incorporating four states for each component considering their level of degradation. Separate models are developed for three cases of corrective repair such perfect, imperfect, and minimal repair with and without condition based opportunistic maintenance. These models are solved using analytical Markov approach. The solutions are validated by the Monte Carlo Simulation. Since the Markov approach is limited to exponential failure and repair rates, the Monte Carlo Simulation based codes can be further extended to accommodate systems which follow non-exponential failure and repair rates.
\end{abstract}

Index Terms-Availability, markov, monte carlo simulation, opportunistic maintenance.

\section{INTRODUCTION}

Maintenance has been defined as a combination of technical and associated administrative actions intended to retain an item or system in, or restore it to, a state in which it can perform its required function [1]. The nascence of modern high technology components has resulted in an increase in complexity and cost of industrial components and systems. This makes it important to maximize the utilization of these components and systems throughout its lifetime. In recent times, the total maintenance cost is largely becoming a substantial portion of the total operating cost of the system or component. Thus the development of a judicious and effective maintenance policy is a major concern for industrial and manufacturing engineers.

Conventional maintenance policies encompass simple corrective and preventive maintenance for components. Corrective maintenance is effectively illustrated by the philosophy "if it isn't broken, don't fix it" [2]. Preventive maintenance, on the other hand, consists of certain scheduled activities that are performed while the system is still functioning. The underlying aim behind preventive maintenance is to reduce the number of unplanned system downtime due to system or component failure.

In the current industrial scenario of increased demand and surfeit global competition, such primitive maintenance policies will not suffice and provide an advantageous edge over competitors. From the maintenance point of view, this advantageous edge can be facilitated by increasing system or

Manuscript received November 2, 2013; revised February 15, 2014.

The authors are with Delhi Technological University, Shahbad Daulatpur, Main Bawana Road, Delhi-110042, India (e-mail: joelprince25@ gmail.com, girish_kumar_ar@yahoo.com). component availability which means reducing downtime without compromising on the system reliability. This has resulted in the adoption of much more efficient maintenance policies such as condition based maintenance and opportunistic maintenance. Opportunistic maintenance can be visualized as a fruitful combination of corrective and preventive maintenance. Opportunistic maintenance makes maximum utilization of the time to perform corrective maintenance on a component or system by using this time to carry out preventive maintenance on components which may require maintenance in the near future. Opportunistic maintenance is not a maintenance policy for a single component, but for a collection of components in a production line or plant [3].

The work in the field of opportunistic maintenance first began by [4]. The authors implemented the opportunistic policy for unmonitored components/systems. Reference [5] shows how this policy was investigated for two components in series, but no mathematical treatment for availability measure was proposed. An imperfect maintenance concept in this context was applied by [6]. Since then, many extensions of opportunistic maintenance have been introduced and studied in the literature. The Markov and Semi-Markov approaches have been used in literature to analyze various maintenance policies. Reference [7] shows how a Markov renewal process was used to construct a maintenance cost model for a simple multi-unit system. A continuous time Markov chain was used by [8] to describe the degradation of a component. A cost model was developed including the presence of resource constraints. Dynamic programming was used by [9] to analyze a condition based and opportunistic model for a two unit deteriorating system involving a cost model. The transient behavior of a multi-unit system was studied by [10] using renewal theory. The instantaneous availability for a fixed time was calculated and a methodology was developed to find times to preventive and opportunistic maintenance such that cost is minimized. His components had binary levels of degradation, i.e. operating or failed.

In the existing literature, the Markov model has been used to analyze systems undergoing various corrective and preventive repairs. The application of Markov for systems undergoing opportunistic maintenance has not been explored so far. Also, the existing literature doesn't take into account multi-state degradation of components and considers components with binary states. The existing literature has resorted to a cost model to analyze and compare various maintenance policies without giving much significance in quantifying the gains of opportunistic maintenance.

This paper overcomes these limitations by firstly developing a system model considering multi-state 
degradation and providing an analytical method based on Markov to analyze the availability. Opportunistic maintenance policies for different types of corrective repairs such as perfect, imperfect, and minimal repairs have been considered. Acknowledging the limitation of the Markov method to be useful for components having exponential failure and repair rates, a Monte Carlo Simulation method is also presented which has been correctly validated by the Markov model to extend this availability analysis to systems having non-exponential failure and repair distributions. The gains of an opportunistic maintenance policy has been quantified in terms of availability of system and this provides us with a suitable decision making tool to choose the best maintenance policy and also compare different combinations of components in terms of availability for a particular maintenance policy.

Software such as RAPTOR (ARNIC, Annapolis, MD), BlockSim (Reliasoft, Tucson, AZ), et al., are widely used for availability analysis of repairable systems. These software programs do not yet have a module for opportunistic maintenance. The proposed methodology can be used as an extension for these software programs.

The remaining paper is organized as follows. Section II will enumerate the approaches used. Section III will describe the system modeling to incorporate multi-level degradation and corrective repair i.e. perfect, imperfect, and minimal, with and without opportunistic maintenance. The solutions of the models based on Markov and Monte Carlo Simulation will be described in Section IV. Section V will use an example to illustrate the methodology, and discuss the results. Finally, Section VI concludes the work.

\section{APPROACHES}

\section{A. Overview of Markov Method}

Markov models are frequently used in reliability and maintainability work where events, such as the failure or repair of the module, can occur at any point in time. The Markov method evaluates the probability of jumping from one known state into the next logical state. This continues until the system being considered has reached the final or totally failed state or until a particular mission time is achieved. The basic assumption of a Markov Process is that the behavior of the system in each state is memory less [11]. For any given system, a Markov model consists of a list of the possible states of that system, the possible transition paths between those states, and the rate parameters of those transitions. Let the symbol $\lambda$ denotes the rate parameter of the transition from State 1 to State 2 and be $y_{i}(t)$ the probability of the system being in State $i$ at time $t$. Fig. 1 shows the Markov model for a simple component with binary states.

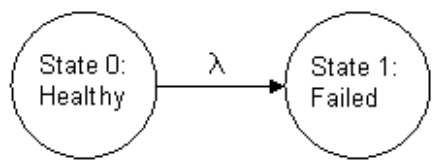

Fig. 1. Markov model for a simple component.

The set of differential equations for this simple model are given by (1), (2) and (3).

$$
\begin{gathered}
\frac{d y_{0}}{d t}=-\lambda y_{\mathrm{o}} \\
\frac{d y_{1}}{d t}=\lambda y_{1} \\
y_{0}+y_{1}=1
\end{gathered}
$$

\section{B. Overview of Monte Carlo Simulation}

The Monte Carlo Simulation is a powerful and versatile tool based on random sampling which has a wide range of applications in various fields. One underlying principle of Monte Carlo Simulation is the law of large numbers, which states that the larger the sample, the more likely the sample mean will be a good estimate of the population mean [12]. The Monte Carlo Simulation can be used to model the availability of systems. It makes use of the failure and repair statistical distributions of components or systems to analyze the system behavior over time [13].

Initially, the simulation time to failure and repair time of the component or system are generated. Random numbers are created using an uniform distribution $(U)$ in the interval $(0,1)$, and a suitable conversion method is used to convert these random numbers into the times for failure and repair. The frequently used conversion methods include the inverse transform method, composition method and acceptance-rejection method. We shall use the inverse transform method in this article due to its simplicity. However, its use is limited to when the repair and failure distributions can be inverted analytically. For an exponential distribution, with parameter $\lambda$, the expression for the random variable $T$ which can be the time to failure or time to repair using an inverse transform method is given by (4).

$$
T=-\frac{1}{\lambda} \ln (1-U)
$$

Also, since the $(1-U)$ is distributed in the same way as $U, T$ can be given by (5).

$$
T=-\frac{1}{\lambda} \ln U
$$

Similarly, for a Weibull distribution with parameters ' $\theta$ ' and ' $\beta$ ' the expression is (6).

$$
T=-\theta(\ln U) \frac{1}{\beta}
$$

After the generation of the failure and repair times of all components or systems according to the appropriate distribution, the sequence of failures and repairs for the system are simulated for a specific mission time. This process is repeated for a large number of simulations. The system availability for a given mission time is calculated as the ratio of the total up time of the system for the total number of simulations to the total of mission time of all of the simulations. 


\section{SYSTEM MODELING}

The system studied in this research is a two component series multi-unit system shown in Fig. 2. The term "unit" could be a component, a set of components, or even a subsystem which can be part of a greater robust or intricate industrial system.

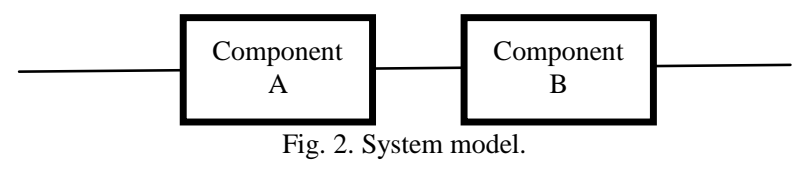

\section{A. Modeling Multi-Level Degradation}

Practically any component/unit which is part of an industrial system undergoes multiple levels of degradation before reaching the failed state. Multi-level degradation, therefore, implies that the component will not have a constant failure rate up to the failed state, but the failure rate will vary as the component reaches the failed state. Each component is assumed to have four levels of degradation with are given by, State 1(Initial State / Good as new), State 2 (Middle State), State 3(Better than worst), and State 4(Failed State).

\section{B. Repair Action}

Since a series system is considered, once a component fails the system will shut down. Corrective repair must be performed on the failed component. The corrective maintenance can be of three types namely perfect, imperfect, or minimal repair. Perfect repair implies that the component is repaired from the 'failed state' to the 'good as new state' (State 1). Imperfect repair implies that the component is repaired from the failed state to the middle state (State 2). Imperfect repair implies a repair of lesser intensity as compared to perfect repair but of a quicker duration. Minimal repair implies that the component is repaired from the failed state to the better than worst state (State 3). Minimal repair is of least intensity and quickest duration. This downtime during the corrective repair process provides an opportunity to perform preventive maintenance on the non-failed component.

\section{Model Development}

The proposed model of two component series system incorporating multi-level degradation can be developed considering the above mentioned multi-level degradation and repair action.

The system contains two components each having a possibility of being in one of the four states at a particular instant of time. Their series combination gives a total of sixteen possible states the entire system can exist throughout its lifetime. This is inclusive of the system state in which both components are in a failed state. In a series system, this state can occur only if both the components fail simultaneously. The probability of this event is considered to be negligible and can be neglected. The generalized assumptions made for the model are as follows.

1) The opportunistic maintenance activity is assumed to be completed before the failed component gets repaired.

2) The component can function perfectly in all states except the failed state.

\section{SOlution TO THE SySTEM Model}

\section{A. Analytical Markov Approach}

Solution of the system model with the analytical Markov approach involves the following steps.

Step 1: Construct a state diagram of the system representing all possible states of the system. The transition from one state to another is specified by an arrow whose direction indicates the direction of transition. Transition rates from one state to another can also be specified in the state diagram by overlaying it with the arrow.

Step 2: Model the differential equation for a particular system corresponding to each state in the similar lines as (1), (2) and (3).

Step 3: Solve the differential equations simultaneously with suitable initial condition and the required mission time. The availability of the system at the specified mission time is calculated by summing the probabilities of the non-failed system states at a particular mission time.

\section{B. Monte Carlo Simulation}

The Monte Carlo Simulation codes which simulate the functioning of the system throughout its mission time are developed, and availability is computed for a large number of trails. The Monte Carlo Simulation codes are based on the following algorithm.

Step 1: Generate random numbers with uniform distribution corresponding to each possible transition of component A and B (failure and repair).

Step 2: Convert these numbers into a value of operating time using the inverse transform method based on the exponential distribution or a non- exponential distribution based on the required condition.

Step 3: At each state compare the times of the possible transition and proceed to the state with the minimum transition time while reducing this time from the transition time of the state not chosen.

Step 4: When a failed state is reached, add the repair time to the system downtime and reset the transition times and the repair time for the failed component.

Step 5: Repeat the steps 1-4 till the cumulative time of the sequence is equal to or more than mission time.

Step 6: Add the downtime for this trail to the total downtime.

Step 7: Repeat steps 5-6 for the required number of trails.

Step 8: Calculate the system availability by dividing the total downtime upon the total mission times after all the trails.

\section{ILLUSTRATION}

A power system consisting of a power transformer (Component A) and a protection and control subsystem for power transformer (Component B) in series was selected for the case study to demonstrate the methodology for availability assessment. The exponential failure and repair distribution is assumed for each subsystem and the data for failure rate and repair rate is taken from the literature [14]. Table I shows the literature values for failure and repair rates of both the components.

To incorporate multi- level degradation in the example, the 
assumption is made that the failure rates of each of the component increases as it deteriorates. This assumption is based on the discussions with experts from the industry who are working in the field of maintenance. The failure and repair rates for different degrading states are obtained by dividing the binary state data available from the literature in consultation with the industry experts. Failure rates $(\lambda)$ are in failures per hour and repair rate $(\mu)$ are in repairs per hour. The superscript and subscript represent the components and the state transition respectively. The respective calculated values are ${ }^{A} \lambda_{12}=1.142 \times 10^{-4},{ }^{A} \lambda_{23}=1.713 \times 10^{-4},{ }^{A} \lambda_{34}=$

$$
3.43 \times \quad 10^{-4}, \quad{ }^{B} \lambda_{12}=1.712 \quad \mathrm{x}
$$$$
10^{-4},{ }^{B} \lambda_{34}=5.136 \times 10^{-4},{ }^{A} \mu_{41}=8.621 \times 10^{-3},{ }^{A} \mu_{42}=17.24 \mathrm{x}
$$$$
10^{-3},{ }^{A} \mu_{43}=34.48 \times 10^{-3},{ }^{B} \mu_{41}=6.76 \times 10^{-2},{ }^{B} \mu_{42}=13.51 \times
$$$$
10^{-2},{ }^{B} \mu_{43}=27.03 \times 10^{-2} \text {. }
$$

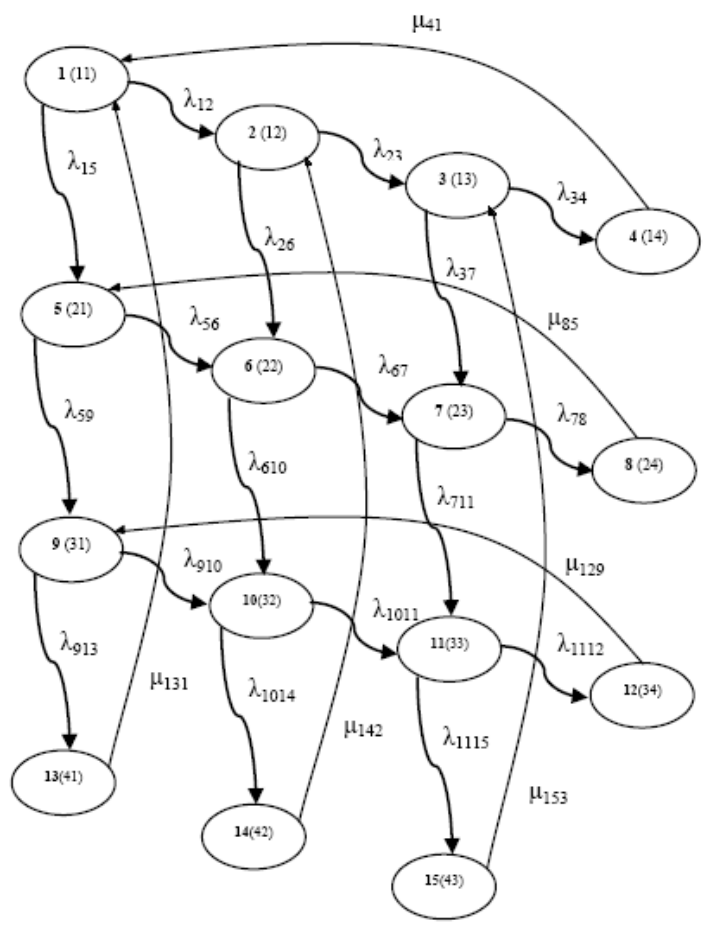

Fig. 3. Corrective Maintenance (Perfect Repair).

Once the transition parameters of failure and repair have been defined the state diagram is drawn. The state diagram for perfect repair is shown in Fig. 3. Fig. 4 shows the state diagram for perfect repair with opportunistic maintenance. Due to space considerations the state diagrams for imperfect and minimal repair with and without opportunistic maintenance are not shown but can be developed along the similar lines. The failure rates and repair rates can also be expressed in terms of system state as $\lambda_{i j}$ or $\mu_{i j}$ where " $i$ " represent the state of the system before transition and " $j$ " represents the state after transition. From Fig. 3 and Fig. 4 it is noticeable that ${ }^{A} \lambda_{12}=\lambda_{15}=\lambda_{26}=\lambda_{37}$. If $y_{i}$ represents the probability of the system being at the state $i$ at the time $t$, then as specified in Section II-A, the set of differential equations are derived for the models shown in Fig. 3 and Fig. 4. The set of equations for Fig. 3 are listed in Appendix I and for Fig. 4 in Appendix II.
TABLE I: LITERATURE VALUES FOR THE COMPONENTS

\begin{tabular}{lllll}
\hline \hline & & & & \\
Component & $\begin{array}{l}\text { Temporary } \\
\text { Failure Rate }\end{array}$ & $\begin{array}{l}\text { Sustained } \\
\text { Failure Rate }\end{array}$ & $\begin{array}{l}\text { Average } \\
\text { Failure Rate }\end{array}$ & $\begin{array}{l}\text { Mean } \\
\text { Repair } \\
\text { Time }\end{array}$ \\
\hline $\begin{array}{l}\text { Power } \\
\begin{array}{l}\text { Transformers } \\
(33-110 \mathrm{kV})\end{array}\end{array}$ & $\begin{array}{l}0.4 /(100 \\
\text { transformers, }\end{array}$ & $\begin{array}{l}0.6 /(100 \\
\text { transformers, } \\
\text { year })\end{array}$ & $\begin{array}{l}0.5 /(100 \\
\text { transformers, }\end{array}$ & $\begin{array}{l}116 \\
\text { hours }\end{array}$ \\
$\begin{array}{l}\text { Protection } \\
\text { and control } \\
\text { for power } \\
\text { transformers }\end{array}$ & $\begin{array}{l}0.9 /(100 \\
\text { transformers, }\end{array}$ & $\begin{array}{l}0.6 /(100 \\
\text { transformers, }\end{array}$ & $\begin{array}{l}0.75 /(100 \\
\text { transformers, }\end{array}$ & 14.8 \\
year) & & year $)$ & year $)$ & \\
\hline \hline
\end{tabular}

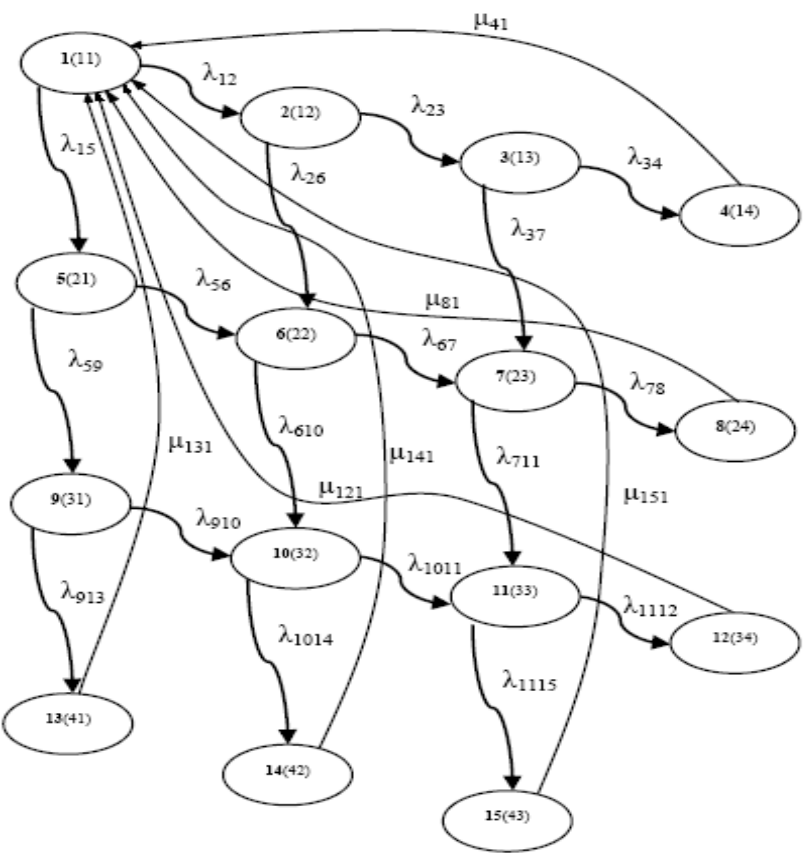

Fig. 4. Corrective maintenance (perfect repair) with opportunistic maintenance.

TABLE II: SYSTEM AVAILABILITY

\begin{tabular}{|c|c|c|c|c|}
\hline $\begin{array}{l}\text { Repair } \\
\text { Action }\end{array}$ & $\begin{array}{c}\text { Availability } \\
\text { without } \\
\text { Opportunisti } \\
\text { c } \\
\text { Maintenance } \\
\text { using } \\
\text { Markov }\end{array}$ & $\begin{array}{c}\text { Availability } \\
\text { without } \\
\text { Opportunisti } \\
\quad \text { c } \\
\text { Maintenance } \\
\text { using Monte } \\
\text { Carlo } \\
\text { Simulation } \\
\end{array}$ & $\begin{array}{c}\text { Availability } \\
\text { with } \\
\text { Opportunisti } \\
\text { c } \\
\text { Maintenance } \\
\text { using } \\
\text { Markov }\end{array}$ & $\begin{array}{c}\text { Availability } \\
\text { with } \\
\text { Opportunisti } \\
\text { c } \\
\text { Maintenance } \\
\text { using Monte } \\
\text { Carlo } \\
\text { Simulation }\end{array}$ \\
\hline $\begin{array}{l}\text { Perfect } \\
\text { Repair }\end{array}$ & 0.99217 & 0.99219 & 0.99483 & 0.99483 \\
\hline $\begin{array}{l}\text { Imperfect } \\
\text { Repair }\end{array}$ & 0.99216 & 0.99216 & 0.9964 & 0.9964 \\
\hline $\begin{array}{l}\text { Minimal } \\
\text { Repair }\end{array}$ & 0.98829 & 0.98889 & 0.99615 & 0.99552 \\
\hline
\end{tabular}

Similarly the set of differential equations can be developed for the cases of imperfect and minimal repairs with and without opportunistic maintenance. The sets of differential equations for each model are solved using MATLAB (Matlab 7.7 2008) with initial conditions as $y_{1}(0)=1, y_{2}(0)=0, y_{3}(0)=0$, $y_{4}(0)=0, y_{5}(0)=0, y_{6}(0)=0, y_{7}(0)=0, y_{8}(0)=0, y_{9}(0)=0, y_{10}(0)=0$, $y_{11}(0)=0, y_{12}(0)=0, y_{13}(0)=0, y_{14}(0)=0, y_{15}(0)=0$ and for larger values mission time to get the steady state results. The system availability is obtained by summing the probabilities of 
operating states. The availability results based on Markov are shown in Table II.

The solution of the model is also obtained using Monte Carlo Simulation Methods. The codes are developed based on the algorithm in section IV-B. The system availability results based on the Monte Carlo Simulation are shown in Table II.

The results clearly show that when the system undergoes opportunistic maintenance there is an increase in the system availability for perfect, imperfect, and minimal repair. This implies that for the given system an opportunistic maintenance policy would increase the availability irrespective of the maintenance action. The Markov and Monte Carlo Simulation give us matching values of system availability. Thus the Monte Carlo Simulation code has been validated and can be further extended to components having a non-exponential failure and repair rates, such as Weibull or Lognormal. Quantifying the gains of opportunistic maintenance using an availability measure helps to identify the maintenance policy resulting in maximum availability. The above results for the illustrative example clearly show that if the system follows an opportunistic maintenance policy with imperfect repair, the system availability will be maximized. This is valid for the current set of data but depending upon the failure and repair rates, the policy may change. This doesn't imply that it is the optimal maintenance policy. The trade-offs in each case should be considered in terms of cost, effect on component life (as an imperfect or minimal repair results in more frequent system failure) and the opportunistic maintenance policy selected must be such that the economic gain due to the increase in system availability must compensate for the cost acquired by performing opportunistic maintenance.

\section{CONCLUSION}

In this article an analytical Markov model is applied to find the system availability of a two component series system with opportunistic maintenance. Separate models are developed for different corrective actions namely perfect, imperfect, and minimal repairs with and without opportunistic maintenance. The results of system availability in all cases are validated using the Monte Carlo Simulation. The Monte Carlo simulation approach can be further extended for components having non-exponential distributions such as Weibull and lognormal distributions. This approach can be used as an extension for the existing software which does not have the opportunistic maintenance module. The suggested approach provides a realistic assessment of availability values of the repairable system due to the incorporation of multi-state degradation. This work can be further extended to more complex systems with more components in various configurations having more levels of degradation. A cost function can also be developed to analyze the economic pros and cons of carrying out opportunistic maintenance. There is a scope for development of a model for an optimal maintenance policy based cost and availability under the limited maintenance resources.

\section{APPENDIX I}

$$
\begin{aligned}
& \frac{d y_{1}}{d t}=-\left(\lambda_{12}+\lambda_{15}\right) y_{1}+\mu_{41} y_{4}+\mu_{131} y_{13} \\
& \frac{d y_{2}}{d t}=-\left(\lambda_{23}+\lambda_{26}\right) y_{2}+\lambda_{12} y_{1}+\mu_{41} y_{4} \\
& \frac{d y_{3}}{d t}=-\left(\lambda_{34}+\lambda_{37}\right) y_{3}+\lambda_{23} y_{2}+\mu_{153} y_{15} \\
& \frac{d y_{4}}{d t}=\lambda_{34} y_{3}-\mu_{41} y_{4} \\
& \frac{d y_{5}}{d t}=-\left(\lambda_{56}+\lambda_{59}\right) y_{5}+\lambda_{15} y_{1}+\mu_{85} y_{8} \\
& \frac{d y_{6}}{d t}=-\left(\lambda_{67}+\lambda_{610}\right) y_{6}+\lambda_{26} y_{2}+\lambda_{56} y_{5} \\
& \frac{d y_{7}}{d t}=-\left(\lambda_{711}+\lambda_{78}\right) y_{7}+\lambda_{67} y_{6}+\lambda_{37} y_{3} \\
& \frac{d y_{8}}{d t}=\lambda_{78} y_{7}-\mu_{85} y_{8} \\
& \frac{d y_{9}}{d t}=-\left(\lambda_{910}+\lambda_{913}\right) y_{9}+\lambda_{59} y_{5}+\mu_{129} y_{12} \\
& \frac{d y_{10}}{d t}=-\left(\lambda_{1011}+\lambda_{1014}\right) y_{10}+\lambda_{910} y_{9}+\lambda_{610} y_{6} \\
& \frac{d y_{11}}{d t}=-\left(\lambda_{1112}+\lambda_{1115}\right) y_{11}+\lambda_{711} y_{7}+\lambda_{1011} y_{10} \\
& \frac{d y_{12}}{d t}=\lambda_{1112} y_{11}-\mu_{129} y_{12} \\
& \frac{d y_{13}}{d t}=\lambda_{913} y_{9}-\mu_{131} y_{13} \\
& \frac{d y_{14}}{d t}=\lambda_{1014} y_{10}-\mu_{142} y_{14} \\
& \frac{d y_{15}}{d t}=\lambda_{1115} y_{11}-\mu_{153} y_{15}
\end{aligned}
$$

\section{APPENDIX II}

$\frac{d y_{1}}{d t}=-\left(\lambda_{12}+\lambda_{15}\right) y_{1}+\mu_{41} y_{4}+\mu_{131} y_{13}$

$+\mu_{81} y_{8}+\mu_{121} y_{12}+\mu_{141} y_{14}+\mu_{151} y_{15}$

$\frac{d y_{2}}{d t}=-\left(\lambda_{23}+\lambda_{26}\right) y_{2}+\lambda_{12} y_{1}$

$\frac{d y_{3}}{d t}=-\left(\lambda_{34}+\lambda_{37}\right) y_{3}+\lambda_{23} y_{2}$

$\frac{d y_{4}}{d t}=\lambda_{34} y_{3}-\mu_{41} y_{4}$

$\frac{d y_{5}}{d t}=-\left(\lambda_{56}+\lambda_{59}\right) y_{5}+\lambda_{15} y_{1}$

$\frac{d y_{6}}{d t}=-\left(\lambda_{67}+\lambda_{610}\right) y_{6}+\lambda_{26} y_{2}+\lambda_{56} y_{5}$

$\frac{d y_{7}}{d t}=-\left(\lambda_{711}+\lambda_{78}\right) y_{7}+\lambda_{67} y_{6}+\lambda_{37} y_{3}$

$\frac{d y_{9}}{d t}=-\left(\lambda_{910}+\lambda_{913}\right) y_{9}+\lambda_{59} y_{5}$ 


$$
\begin{aligned}
& \frac{d y_{10}}{d t}=-\left(\lambda_{1011}+\lambda_{1014}\right) y_{10}+\lambda_{910} y_{9}+\lambda_{610} y_{6} \\
& \frac{d y_{11}}{d t}=-\left(\lambda_{1112}+\lambda_{1115}\right) y_{11}+\lambda_{711} y_{7}+\lambda_{1011} y_{10} \\
& \frac{d y_{12}}{d t}=\lambda_{1112} y_{11}-\mu_{121} y_{12} \\
& \frac{d y_{13}}{d t}=\lambda_{913} y_{9}-\mu_{131} y_{13} \\
& \frac{d y_{14}}{d t}=\lambda_{1014} y_{10}-\mu_{141} y_{14} \\
& \frac{d y_{15}}{d t}=\lambda_{1115} y_{11}-\mu_{151} y_{15}
\end{aligned}
$$

\section{REFERENCES}

[1] Collection and exchange of reliability and maintenance data for equipment, ISO 14224, 2004.

[2] J. Huang, "Preventive maintenance program development for multi-unit system with economic dependency- stochastic modeling and simulation study," Ph.D. Dissertation, Dept. of Industrial and Management Systems, University of South Florida, University of South Florida, Tampa, 1993.

[3] R. P. Nicolai and R. Dekker, "Optimal maintenance of multi-component systems: A review," K. A. H. Kobbacy and D. N. P. Murthy, eds, London, 2008.

[4] R. Radner and D. W. Jorgenson, "Opportunistic replacement of a single part in the presence of several monitored parts," Management Science, vol. 10, issue 1, pp. 70-84, 1963.

[5] F. A. V. D. D. Schouten and S. G. Vanneste, "Analysis and computation of $(\mathrm{n}, \mathrm{N})$ strategies for maintenance of a two component system," European Journal of Operational Research, vol. 48, issue 2, pp. 260-274, 1990.

[6] H. Wang, H. Pham, and A. E. Izundu, "Optimal preparedness maintenance of multi-unit systems with imperfect maintenance and economic dependence," Recent advances in reliability and quality engineering. New Jersey: World Scientific, pp. 75-92, 2001.

[7] B. Castanier, A Grall, and C. Berenguer, "A condition-based maintenance policy with non-periodic inspections for a two-unit series system," Reliability Engineering and System Safety, vol. 87, pp. 109-120, January 2005.
[8] S. Ambani, L. Li, and J. Ni, "Condition-Based maintenance decision-making for multiple machine systems," Journal of Manufacturing Science and Engineering, vol. 131, pp. 0310091-0310099, 2009.

[9] Z. Zhang, S. Wu, and B. Li, "A condition-based and opportunistic maintenance model," in Proc. International Conference on Quality, Reliability, Risk, Maintenance, and Safety Engineering, pp. 590 - 595, Xi'an, 2011.

[10] S. Jain, "Opportunistic maintenance policy of a multi-unit system under transient state," M.S. Thesis, Dept. of Industrial and Management Systems, University of South Florida, Tampa, Florida, 2005.

[11] Relex Software Technical Brief. Markov Analysis Accurately Models Dynamic Behaviors. [Online]. Available: www.relexsoftware.com/reliability/brief/markov_brief1.asp

[12] MIL-HDBK-338B, Military Handbook, Electronic Reliability Design Handbook, Washington, DC, USA: United States Department of Defense, 1998

[13] A. Dennis, "Application of Monte Carlo simulation system reliability analysis," presented at the 20th International Pump Users Symposium, Houston, Texas, March 17-20, 2003.

[14] F. Roos and S. Lindahl, "Distribution system component failure rates and repair times - an overview," presented at the Nordic Distribution and Asset Management Conference, Espoo, Finland, August 23-24, 2004.

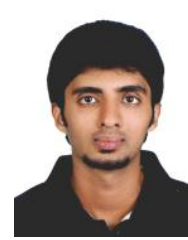

Joel P. Varghese is a third year engineering student. $\mathrm{He}$ is pursuing his bachelor of technology degree in mechanical engineering at Delhi Technological University, Delhi, India.

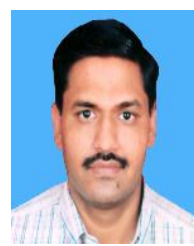

Girish Kumar is presently an assistant professor in Mechanical Engineering Department at Delhi Technological University, Delhi, India. He is also pursuing his doctoral degree from the Indian Institute of Technology, Delhi, India. His current research interest is in the RAM modeling and analysis of repairable mechanical systems. 